\title{
Biaxial Order Parameter in the Homologous Series of Orthogonal Bent-Core Smectic Liquid Crystals
}

\author{
S. Sreenilayam \\ Technological University Dublin \\ Yuri Panarin \\ Technological University Dublin, yuri.panarin@tudublin.ie \\ J. K. Vij \\ Trinity College Dublin, Ireland, jvjj@tcd.ie
}

See next page for additional authors

Follow this and additional works at: https://arrow.tudublin.ie/engscheleart2

Part of the Electrical and Computer Engineering Commons

\section{Recommended Citation \\ Lehmann, A. et al. (2013). Biaxial order parameter in the homologous series of orthogonal bent-core smectic liquid crystals. Physical Review E, vol. 88, pp 1-5. DOI:10.1103/PhysRevE.88.012504}

This Article is brought to you for free and open access by the School of Electrical and Electronic Engineering at ARROW@TU Dublin. It has been accepted for inclusion in Articles by an authorized administrator of ARROW@TU Dublin. For more information, please contact arrow.admin@tudublin.ie, aisling.coyne@tudublin.ie, gerard.connolly@tudublin.ie.

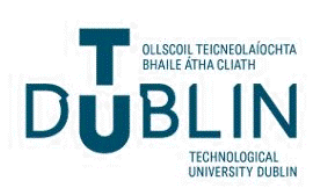




\section{Authors}

S. Sreenilayam, Yuri Panarin, J. K. Vij, M. Osipov, A. Lehmann, and C. Tschierske

This article is available at ARROW@TU Dublin: https://arrow.tudublin.ie/engscheleart2/162 


\title{
Biaxial order parameter in the homologous series of orthogonal bent-core smectic liquid crystals
}

\author{
S. Sreenilayam, ${ }^{1}$ Y. P. Panarin,,${ }^{1,2, *}$ J. K. Vij,${ }^{2,5,}{ }^{\dagger}$ M. Osipov, ${ }^{3}$ A. Lehmann, ${ }^{4}$ and C. Tschierske ${ }^{4}$ \\ ${ }^{1}$ School of Electrical and Electronic Engineering, Dublin Institute of Technology, Dublin 8, Ireland \\ ${ }^{2}$ Department of Electronic and Electrical Engineering, Trinity College, University of Dublin, Dublin 2, Ireland \\ ${ }^{3}$ Department of Mathematics, University of Strathclyde, Glasgow G1 1XH, United Kingdom \\ ${ }^{4}$ Institute of Chemistry, Organic Chemistry, Martin-Luther-University Halle-Wittenberg D06120, Germany \\ ${ }^{5}$ School of Electronic and Electrical Engineering, Sungkyunkwan University, Suwon 440-476, Korea
}

(Received 13 February 2013; published 11 July 2013)

\begin{abstract}
The fundamental parameter of the uniaxial liquid crystalline state that governs nearly all of its physical properties is the primary orientational order parameter $(S)$ for the long axes of molecules with respect to the director. The biaxial liquid crystals (LCs) possess biaxial order parameters depending on the phase symmetry of the system. In this paper we show that in the first approximation a biaxial orthogonal smectic phase can be described by two primary order parameters: $S$ for the long axes and $C$ for the ordering of the short axes of molecules. The temperature dependencies of $S$ and $C$ are obtained by the Haller's extrapolation technique through measurements of the optical birefringence and biaxiality on a nontilted polar antiferroelectric $\left(\mathrm{Sm}-A P_{A}\right)$ phase of a homologous series of LCs built from the bent-core achiral molecules. For such a biaxial smectic phase both $S$ and $C$, particularly the temperature dependency of the latter, are being experimentally determined. Results show that $S$ in the orthogonal smectic phase composed of bent cores is higher than in Sm- $A$ calamatic LCs and $C$ is also significantly large.
\end{abstract}

DOI: 10.1103/PhysRevE.88.012504

PACS number(s): $61.30 . \mathrm{Gd}, 77.84 .-\mathrm{s}, 83.80 . \mathrm{Xz}$

\section{INTRODUCTION}

Liquid crystals (LCs) are highly anisotropic complex fluids and their physical properties, such as optical and dielectric and material parameters including elastic and viscosity coefficients, etc., can be expressed in terms of the order parameters. The number of order parameters depends on the phase symmetry; their magnitudes may depend on their definition. Maier-Saupe [1] proposed a molecular field theory for a uniaxial nematic mesophase in which the alignment of molecules along a preferred axis is expressed in terms of the scalar order parameter $S$, where $S=\frac{1}{2}\left\langle\cos ^{2} \theta-1\right\rangle, \theta$ is the angle between the long molecular axis and the director, and $\langle(\ldots)\rangle$ denotes the ensemble average. Meanwhile de Gennes and Prost [2] noticed that even macroscopically uniaxial LC phases generally consist of biaxial shaped molecules and the molecular biaxiality affects the general form of the orientational tensor order parameters.

The thermotropic biaxial nematic phase was first theoretically predicted by Freiser [3] in 1970, who generalized the Maier-Saupe molecular field theory by including two molecular second-rank tensor order parameters for a lower symmetry system. These tensors can be diagonalized using a frame that coincides with the directors of a biaxial nematic phase. This diagonalization made by Straley [4] gave rise to four Straley scalar order parameters [5,6]. In this work, Straley assumed a simplified molecular shape with the symmetry of a rectangular parallelepiped rather than applying the theory to an object of arbitrary shape. The main result of this work is that such systems undergo phase transitions from the isotropic to the biaxial $N_{B}$ phase through the uniaxial nematic phase,

\footnotetext{
*yuri.panarin@ dit.ie

†jvij@tcd.ie
}

either calamitic $N_{U}$ or discotic $N_{D}$, depending on the sign of the molecular shape biaxiality. This model is used as the basis for the follow on of theoretical and simulation works. A potential existence of the biaxial nematic phase for other biaxial molecular particles has been supported by a number of computer simulations [7-10], which were recently summarized in Ref. [11].

In spite of significant progress in theoretical and computational physics made so far, the experimental studies on low molar mass thermotropic biaxial nematics could not unambiguously find a biaxial nematic phase as yet. The optical biaxiality has been found to be too low to ascribe it to the inherent biaxiality of the nematic phase after the effects of surfaces and other experimental errors are taken into account. Furthermore there are problems associated with the independent alignment of long and short molecular axes in biaxial nematics along the two orthogonal directions.

In contrast to the nematics, relatively large biaxiality was observed in 2001 in the orthogonal Sm-A-like phase of LCs exhibited by bent-core shaped molecules. Since the molecules are constrained to lie in layers, there are no problems associated with the independent alignment of the two axes along the two orthogonal directions.

Essentially two different types of biaxial smectic phases were discovered: polar biaxial (Sm- $\left.C_{P A}\right)$ [12] in 2001 and nonpolar biaxial $\left(\mathrm{Sm}-A_{d b}\right)$ [13] [here $d$ stands for double layer (interdigitated) and $b$ for biaxial] in 2002. Sm- $C_{P A}$ is composed of orthogonal polar smectic layers with antiferroelectric structure, i.e., alternating polar directions in the neighboring layers are antiferroelectrically arranged. Such a structure was also observed in a homologous series of an LC compound [14] and $\mathrm{Sm}-C_{P A}$ has been renamed as $\mathrm{Sm}-A P_{A}$, where $\mathrm{Sm}-A$ stands for orthogonal (no tilt) smectics, $P$ for polar layers, and subscript $A$ for antiferroelectric. In the $\mathrm{Sm}-A P_{A}$ phase, polar directions in the neighboring layers are antiparallel 
leading to the biaxial antiferroelectric phase. An application of the in-plane electric field higher than its threshold value in the $\mathrm{Sm}-A P_{A}$ phase causes a field-induced transition to the ferroelectric $\mathrm{Sm}-A P_{F}$ state $[15,16]$.

In addition to the antiferroelectric $\mathrm{Sm}-A P_{A}$ phase, several different polar biaxial phases such as macroscopically uniaxial Sm- $A P_{R}$ [17] and Sm- $A P_{A R}$ [18], and biaxial ferroelectric $\mathrm{Sm}-A P_{F}$ [19], all in their ground states, were discovered. The common characteristic of these three phases is that they are nontilted (Sm- $A$-like) and possess long-range or shortrange order of the in-layer polarizations in smectic layers. In these three phases, the molecular rotation around the major director within a smectic layer is biased at least to some extent. Sm- $A P_{R}$ macroscopically is a uniaxial phase with a random distribution of the polar directors (or the in-layer spontaneous polarizations) in smectic layers. The Sm- $A P_{A R}$ phase was initially proposed [18] to consist of local antiferroelectric orderings. Later on it was found to have a short pitch of a three-layer structure, supported by modeling of the experimental dependence of biaxiality on the applied electric field [20]. The ferroelectric $\mathrm{Sm}-A P_{F}$ which exists in the absence of an external in-plane field is a biaxial phase with a typical ferroelectric structure in the adjacent layers.

\section{THEORY}

In this paper, we present theoretical and experimental studies of orientational order parameters for smectic LCs composed of achiral bent-core molecules by the measurements of biaxiality and birefringence. The biaxiality in orthogonal smectics can be exploited to produce fast electro-optic devices since it has already been established that LCs are excellently aligned in these phases $[15,16]$. The anisotropic properties of LCs can be characterized by the molecular polarizability tensor $\chi_{n, i j}^{M}$ which can be diagonalized in the molecular frame as

$$
\chi_{n, i j}^{M}=\chi_{33} a_{i} a_{j}+\chi_{22} b_{i} b_{j}+\chi_{11} c_{i} c_{j},
$$

where the orthogonal unit vectors $\boldsymbol{a}, \boldsymbol{b}$, and $\boldsymbol{c}$ are the principal molecular axes and $\chi_{11}, \chi_{22}, \chi_{33}$ are the principal values of the polarizability tensor.

The components of the average polarizability depend on the orientational order parameters and can be conveniently calculated by rewriting the average polarizability tensor as a sum of orthogonal contributions:

$$
\begin{aligned}
\chi_{i j} & =\left\langle\chi_{n, i j}^{M}\right\rangle \\
& =\bar{\chi} \delta_{i j}+\Delta \chi\left\langle\left(a_{i} a_{j}-\frac{1}{3} \delta_{i j}\right)\right\rangle+\chi_{\perp}\left\langle\left(b_{i} b_{j}-c_{i} c_{j}\right)\right\rangle,
\end{aligned}
$$

where $\bar{\chi}=\left(\chi_{11}+\chi_{22}+\chi_{33}\right) / 3$ is the average molecular polarizability, $\Delta \chi=\chi_{33}-\left(\chi_{11}+\chi_{22}\right) / 2$ is the polarizability anisotropy, and $\chi_{\perp}=\chi_{22}-\chi_{11}$ is the biaxiality of the molecular polarizability, and where $\langle(\ldots)\rangle$ denotes the statistical average.

In an orthogonal biaxial smectic phase, the averages of the two molecular tensors $\left\langle\left(a_{i} a_{j}-\frac{1}{3} \delta_{i j}\right)\right\rangle$ and $\left\langle\left(b_{i} b_{j}-c_{i} c_{j}\right)\right\rangle$ give rise to the four orientational order parameters (see, for example, [4,21]):

$$
\begin{aligned}
& \left\langle\left(a_{i} a_{j}-\frac{1}{3} \delta_{i j}\right)\right\rangle=S\left(n_{i} n_{j}-\frac{1}{3} \delta_{i j}\right)+\frac{1}{2} P\left(m_{i} m_{j}-h_{i} h_{j}\right), \\
& \left\langle\left(b_{i} b_{j}-c_{i} c_{j}\right)\right\rangle=D\left(n_{i} n_{j}-\frac{1}{3} \delta_{i j}\right)+\frac{1}{2} C\left(m_{i} m_{j}-h_{i} h_{j}\right),
\end{aligned}
$$

where the unit vector $\boldsymbol{n}$ is the primary director and $\boldsymbol{m}$ and $\boldsymbol{h}$ are the secondary directors of the biaxial phase. Here $S$ is the uniaxial nematic order parameter, $D$ is the secondary uniaxial parameter which exists also in the uniaxial nematic phase, $P$ specifies the biaxial ordering of long molecular axes in the biaxial phase, and $C$ is the primary biaxial order parameter of short molecular axes.

The scalar order parameters $S, P, D$, and $C$ as defined by Straley are expressed as the averages [4,21]: $S=\left\langle P_{2}(\cos \theta)\right\rangle$, $P=\left\langle\sin ^{2} \theta \cos 2 \phi\right\rangle, D=\frac{3}{2}\left\langle\sin ^{2} \theta \cos 2 \psi\right\rangle$, and $C=\frac{1}{2}\langle(1+$ $\left.\left.\cos ^{2} \theta\right) \cos 2 \phi \cos 2 \psi-2 \cos \theta \sin 2 \varphi \sin 2 \psi\right\rangle$, where $\theta$ is the polar angle between the primary axis $\boldsymbol{a}$ and the director $\boldsymbol{n}$, and $\phi$ and $\psi$ are the remaining two Euler angles which specify the orientation of a biaxial molecule. $\phi$ is the azimuthal angle which specifies the orientation of the projection of the axis $\boldsymbol{a}$ on the $(\boldsymbol{m}, \boldsymbol{h})$ plane, and $\psi$ specifies the angle between the short axis $\boldsymbol{b}$ and the $(\boldsymbol{a}, \boldsymbol{n})$ plane.

One can notice that the order parameters $P$ and $D$ vanish in the limit $S \rightarrow 1$ and thus they can be neglected in the case of high $S$. Then one obtains the following approximate expressions for the principal values of the average molecular polarizability:

$$
\begin{aligned}
& \chi_{n}^{M}=\bar{\chi}+\frac{2}{3} \Delta \chi S, \\
& \chi_{m}^{M} \approx \bar{\chi}-\frac{1}{3} \Delta \chi S+\chi_{\perp} C, \\
& \chi_{h}^{M} \approx \bar{\chi}-\frac{1}{3} \Delta \chi S-\chi_{\perp} C .
\end{aligned}
$$

Finally the biaxiality of the average polarizability tensor can be expressed as $\chi_{m}-\chi_{h} \approx 2 \chi_{\perp} C$. Therefore the primary biaxial order parameter can be determined as

$$
C \approx \frac{\chi_{m}-\chi_{h}}{2 \chi_{\perp}} \approx \frac{1}{2} \frac{\chi_{m}-\chi_{h}}{\chi_{22}-\chi_{11}} .
$$

One may notice that according to de Gennes [2], the biaxial order parameter may in principle be related to the biaxiality of any physical quantity including the difference between the refractive indices $n_{m}-n_{h}$. On the other hand, it is important to establish a relationship between $n_{m}-n_{h}$ and the orientational order parameters defined by Eqs. (3) and (4) based on molecular tensors. One can use the equations $n_{n}^{2}=$ $1+4 \pi \chi_{n}, n_{m}^{2}=1+4 \pi \chi_{m}, n_{h}^{2}=1+4 \pi \chi_{h}$, where $n_{n}, n_{m}, n_{h}$ are the principal refractive indices of the biaxial phase and $\chi_{n}, \chi_{m}, \chi_{h}$ are the principal values of the macroscopic dielectric susceptibility. The macroscopic susceptibility tensor can be written in the form $\chi=\rho f \cdot \chi^{M}$ where $\rho$ is the molecular number density, $f$ is the local field tensor, and $\chi^{M}$ is the average molecular polarizability tensor, given by Eq. (5). In the characterization of LCs one often uses the simple semiempirical Vuks formula [22] for the local field tensor $f=\left(\varepsilon_{\mathrm{av}}+2\right) / 3$ where $\varepsilon_{\mathrm{av}}$ is the average dielectric constant. In this approximation the refractive indices are directly

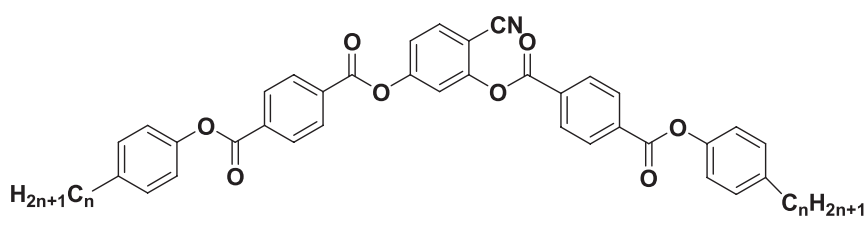

FIG. 1. Chemical structure of a banana shaped molecule of bent-core LC compounds under investigation. 
TABLE I. Phase sequence and the phase transition temperatures (deg C) of the homologous series of achiral smectic LCs composed of banana shaped molecules, obtained on cooling from the isotropic temperature.

\begin{tabular}{lc}
\hline \hline Chain length & Phase sequence \\
\hline 8 & $\mathrm{Sm}-A P_{A} 121{ }^{\circ} \mathrm{C} \mathrm{Sm}-A 163^{\circ} \mathrm{C} \mathrm{I}$ \\
12 & $\mathrm{Sm}-A P_{A} 111^{\circ} \mathrm{C} \mathrm{Sm}-A P_{R} 158^{\circ} \mathrm{C} \mathrm{Sm}-A 164^{\circ} \mathrm{C} \mathrm{I}$ \\
14 & $\mathrm{Sm}-A P_{A} 91^{\circ} \mathrm{C} \mathrm{Sm}-A P_{A R} 108^{\circ} \mathrm{C} \mathrm{Sm}-A P_{R} 111^{\circ} \mathrm{C} \mathrm{Sm}-A 164{ }^{\circ} \mathrm{C} I$ \\
\hline \hline
\end{tabular}

expressed in terms of the components of the average molecular polarizability which depend on the order parameters $S$ and $C$.

In this paper we use the Vuks formulas [22] to determine the order parameters based on the measurements of the birefringence and biaxiality of the refractive indices. Expanding $n_{n}, n_{m}, n_{h}$ in $S$ and $C$ to the first order one obtains the following approximate expressions for the order parameters:

$$
\begin{aligned}
S & \approx \frac{n_{n}-\left(n_{m}+n_{h}\right) / 2}{n_{33}-\left(n_{22}+n_{11}\right) / 2}=\frac{\Delta n}{\Delta n_{0}}, \\
C & \approx \frac{1}{2} \frac{n_{m}-n_{h}}{n_{22}-n_{11}}=\frac{1}{2} \frac{\delta n}{\delta n_{0}},
\end{aligned}
$$

where $\Delta n$ and $\delta n$ are the measured values of birefringence and biaxiality while $\Delta n_{0}$ and $\delta n_{0}$ are the corresponding maximum values which refer to the system of perfectly ordered molecules.

Kuczynski et al. [23] suggested an approximate empirical formula for normalization of the optical birefringence similar to that used by Haller [24] for polarizabilities. It has been shown that the nematic order parameter obtained from the optical anisotropy is equal to the one evaluated from magnetic polarizability anisotropy with the accuracy of $\pm 5 \%$.

In this extrapolation one assumes that for temperatures far enough from the clearing point, the expression $\log \Delta \chi(T)$ [23] is a linear function of the logarithm of the reduced temperature. On the other hand, one postulates that the order parameter is equal to 1 at the absolute zero of temperature which means that all molecules are parallel to each other, i.e., $\lim _{T \rightarrow 0}[\log (\Delta n)]=\log \Delta n_{0}$.

On applying these postulates the birefringence can be rewritten as [23]

$$
\begin{aligned}
\Delta n(T) & =\Delta n_{0}\left(1-\frac{T}{T^{*}}\right)^{\beta} \text { or } \\
\log [\Delta n(T)] & =\log \Delta n_{0}+\beta \log \left(1-\frac{T}{T^{*}}\right),
\end{aligned}
$$

where $T$ is the absolute temperature, and $T^{*}$ and $\beta$ and are constants. Similarly,

$$
\begin{aligned}
\delta n(T) & =\delta n_{0}\left(1-\frac{T}{T^{*}}\right)^{\beta} \text { or } \\
\log [\delta n(T)] & =\log \delta n_{0}+\beta \log \left(1-\frac{T}{T^{*}}\right) .
\end{aligned}
$$

\section{EXPERIMENT}

Measurements of biaxiality have been carried out on a bentcore 4-cyanoresorcinol bisbenzoate with two terephthalatebased wings [25], with alkyl chain lengths of 8,12 , and 14. The chemical structure of these compounds is given in Fig. 1, and Table I lists the phase sequence and the phase transition temperatures, on cooling from the isotropic temperature. All compounds of these homologous series exhibit an orthogonal biaxial antiferroelectric $\mathrm{Sm}-A P_{A}$ phase in the lower temperature range.

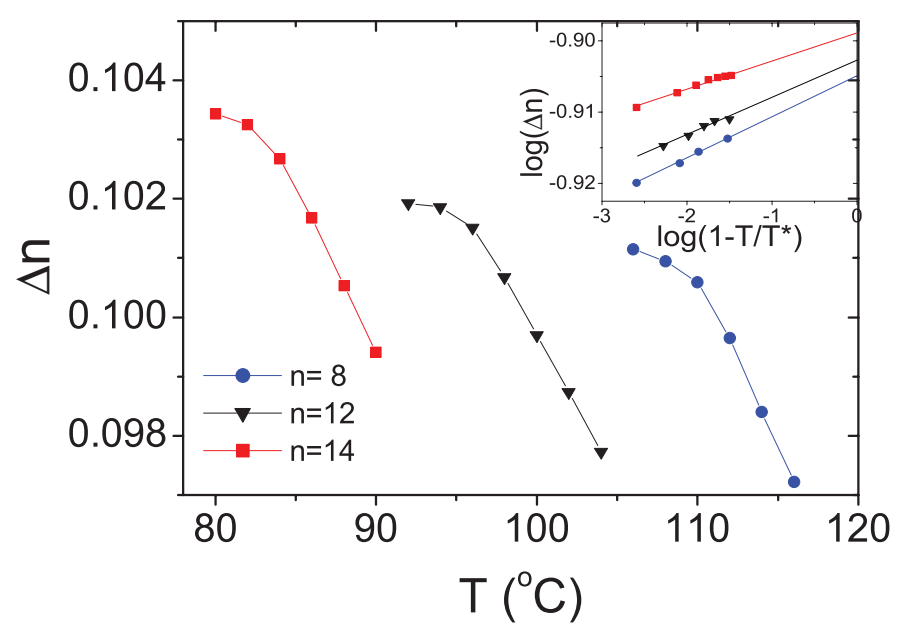

(a)

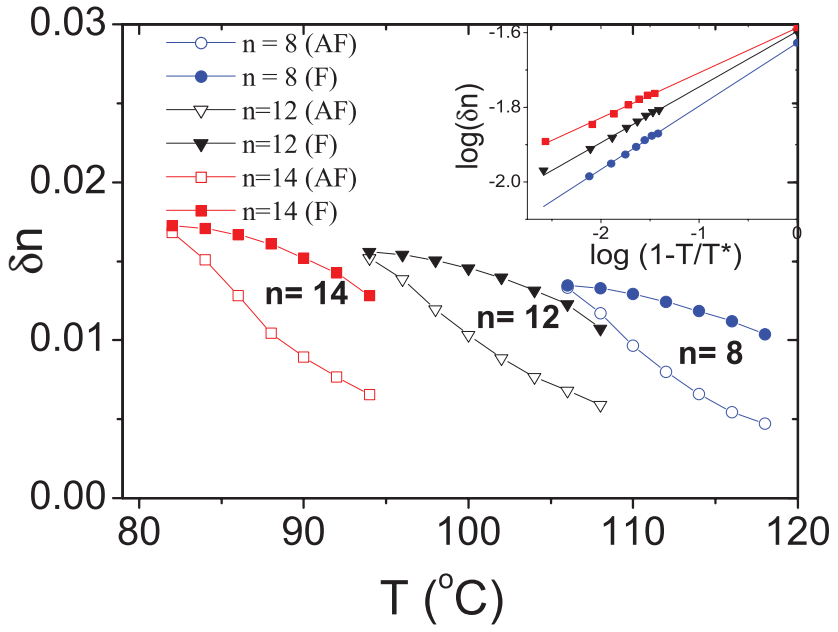

(b)

FIG. 2. (Color online) Temperature dependencies of (a) optical birefringence, $\Delta n$, and (b) biaxiality, $\delta n$, in undisturbed Sm- $A P_{A}$ phase (open symbols) and in the field-induced $\mathrm{Sm}-A P_{F}$ state (filled symbols) for three studied compounds. 
Two different types of $\sim 7-\mu$ m-thick cells were used in optical measurements: the homogeneous cells with planar alignment for birefringence $(\Delta n)$ measurements and homogeneous cells with homeotropic alignment for biaxiality $(\delta n)$ measurements. The planar cells were built from two transparent indium tin oxide (ITO) glass substrates coated by RN1175 polymer (Nissan Chemicals, Japan) layer. The electrodes for homeotropic cells were made by etching an $\sim 80$ - $\mu \mathrm{m}$-wide stripe on the ITO surface to apply an in-plane electric field. The homeotropic alignment was obtained by treating the glass plates with a polymer, AL60702 (JSR, Korea). The tilting optical compensator technique was used to measure the magnitude of biaxiality. The cells were placed in the temperature controlled hot stage between crossed polarizers of the polarizing microscope, at the angle $\alpha=45^{\circ}$ between the main optical axis and polarizer's axis. The optical compensator based on a rotating birefringent plate was a standard accessory for a Leitz polarizing microscope (Leitz Wetzlar, Germany 0345), built according to the Nikitin-Berek principle. This optical compensator enables one to measure extremely small optical retardation of a particular domain and it helps to avoid the influence of any kind of defects within the cell. The optical retardation is measured as a function of voltage and temperature by inserting a tilting compensator plate in the slot of the polarizing optical microscope.

The effect of electric field on biaxiality in the $\mathrm{Sm}-A P_{A}$ phase was studied in greater detail in Ref. [16]. An application of electric field to biaxial $\mathrm{Sm}-A P_{A}$ disturbs an antiparallel structure of polar directors in adjacent layers and finally causes the phase transition to the field-induced biaxial ferroelectric state $\mathrm{Sm}-A P_{F}$ through the intermediate nonbirefringent uniaxial state [15]. Theoretically both states must be optically equivalent, i.e., have the same polarizability tensor but preliminary experiment shows that the birefringence magnitudes in Sm$A P_{A}$ and in the field-induced Sm- $A P_{F}$ phases are of the same value $\left(\Delta n_{A F} \approx \Delta n_{F}\right)$ while values of biaxiality are different $\left(\delta n_{A F}<\delta n_{F}\right)$ [26]. Therefore the measurements of biaxiality were performed in both the undisturbed antiferroelectric state and in the field-induced ferroelectric state.

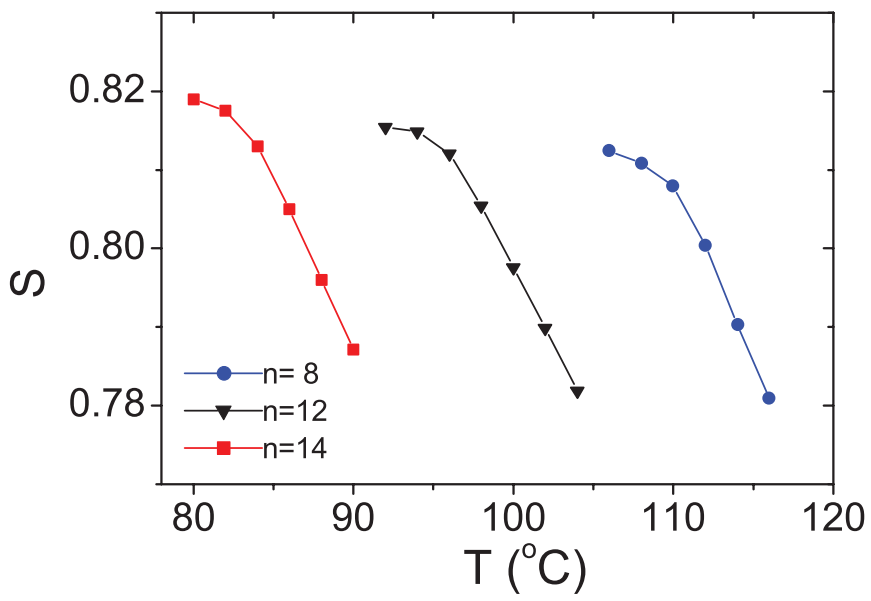

(a)
TABLE II. The maximal values of biaxiality $\delta n_{0}$ and birefringence $\Delta n_{0}$ deduced from extrapolation to $0 \mathrm{~K}$.

\begin{tabular}{lcc}
\hline \hline Chain length & $\Delta n_{0}$ & $\delta n_{0}$ \\
\hline 8 & 0.1245 & 0.0236 \\
12 & 0.1250 & 0.0253 \\
14 & 0.1263 & 0.0258 \\
\hline \hline
\end{tabular}

\section{RESULTS AND DISCUSSION}

Figure 2 shows the temperature dependence of birefringence [Fig. 2(a)] and biaxiality [Fig. 2(b)] in three different compounds for both $\mathrm{Sm}-A P_{A}$ and field-induced $\mathrm{Sm}-A P_{F}$ phases. Both biaxiality and birefringence grow on cooling, as expected, indicating a gradual increase of the order parameters. Figure 2(b) shows that the magnitude of biaxiality of antiferroelectric state is smaller than the field-induced ferroelectric state $\left(\delta n_{A F}<\delta n_{F}\right)$, especially in the higher temperature range.

On cooling, the biaxiality $\delta n_{A F}$ increases faster than $\delta n_{F}$ and finally, at a particular lower temperature, the biaxialities of both antiferroelectric and ferroelectric states become almost equal $\left(\delta n_{F} \approx \delta n_{A F}\right)$ in all of the three studied compounds. Therefore the $\mathrm{Sm}-A P_{A}$ phase in the bent-core compounds shows a weak antiferroelectric interaction which is supported by high intensity of the dielectric mode [12] and large fieldinduced spontaneous polarization [16].

The experimental data were fitted to Eqs. (9) and (10) (Fig. 2, inset) and extrapolated to the absolute zero of temperature $(T=0 \mathrm{~K})$ where (i) the order parameter is assumed to be unity and $\log (\delta n)$ is a linear function of the logarithm of the reduced temperature $\left[\left(T^{*}-T\right) / T^{*}\right]$ for the points far enough from the clearing temperature $\left(T^{*}\right)$ (see Fig. 2, inset). The maximal (extrapolated to $0 \mathrm{~K}$ ) values of biaxiality $\delta n_{0}$ and birefringence $\Delta n_{0}$ for all three studied compounds are listed in Table II.

One may note that both maximal (extrapolated) birefringence $\Delta n_{0}$ and biaxiality $\delta n_{0}$ slightly increase with the alkyl chain elongation; nevertheless the difference between them

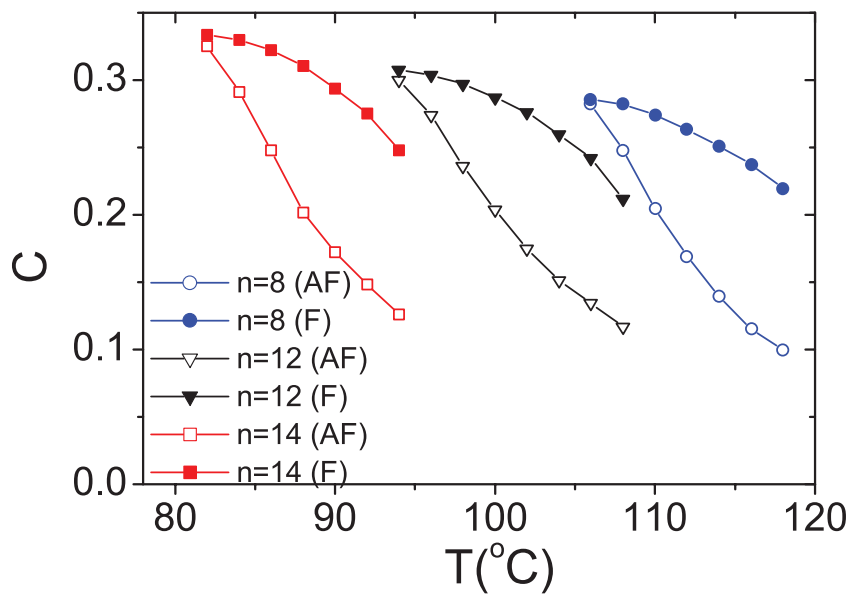

(b)

FIG. 3. (Color online) Temperature dependencies of primary order parameters for uniaxial $S$ (a) and for biaxial $C$ (b) in both undisturbed and field-induced $\mathrm{Sm}-A P_{F}$ states; undisturbed open symbols and field-induced (filled symbols). 
is just within the experimental error mainly due to limited accuracy in the measurement of cell thicknesses, which was $\pm 3 \%$ for planar cells and $\pm 5 \%$ for homeotropic cells.

Figure 3 shows the temperature dependence of primary uniaxial order parameter $S$ (a) and the primary biaxial order parameter $C$ (b) calculated from the temperature dependencies of birefringence and biaxiality (Fig. 2) and using extrapolated values of birefringence $\Delta n_{0}$ and biaxiality $\delta n_{0}$ from Eqs. (7) and (8).

It should be noted that only the values of the biaxial order parameter $C$, obtained in the field-induced $\mathrm{Sm}-A P_{F}$ states [filled symbols in Fig. 3(b)], have a direct physical meaning while the open symbols in Fig. 3(b) represent some apparent values which are affected by other types of disorder. One may assume that the alignment of the second director in the homeotropic cell is not perfect and this improves with decreasing temperature. In addition, both antiferro- and ferroelectric phases (states) are characterized by additional polar order parameters which also increase with decreasing temperature and may contribute to the birefringence in the second approximation. This explains why both the measured birefringence and the apparent biaxial order parameter in the undisturbed Sm$A P_{A}$ phase increase faster than linear with decreasing temperature. Application of the electric field improves the alignment of the second director and drives the polar order parameter towards saturation but only weakly affects the nonpolar parameter $C$. As a result the temperature variation of measured order parameter in the field-induced phase has a normal shape which represents the actual variation of the biaxial order.

\section{CONCLUSION}

In summary, we have developed an experimental method to estimate primary order parameters for uniaxial and biaxial states from the temperature dependent measurements of the optical birefringence and biaxiality using Haller's extrapolation approach. We have applied this technique to three compounds of orthogonal $\mathrm{Sm}$ - $A$-like bent-core homologous series, with $n=8,12,14$ carbon atoms in the alkyl chain. All these compounds show at least the $\mathrm{Sm}-A P_{A}$ phase, in which the optical measurements were performed. Both birefringence and biaxiality slightly increase with the alkyl chain elongation, while both order parameters $S$ and $C$ show the opposite tendency.

The absolute values of the primary uniaxial order parameter $S$ in the bent-core LCs are higher than in conventional Sm- $A$ phases which can be explained by strong polar interactions of bent molecular cores. The biaxial order parameters in the range of $0.25-0.35$ (or $0.5-0.7$ when normalized to unity [4]) are smaller than uniaxial order parameter as expected, but still rather high compared to the other biaxial systems. This is supported by the theoretical model developed in Ref. [21]. The authors show that the board shape symmetry is not sufficient to provide a macroscopic biaxiality, while the lower molecular symmetries such as $C_{2 h}$ in tetrapodes and $C_{2 v}$ in bent-core molecules are required.

\section{ACKNOWLEDGMENTS}

The authors thank the DIT research program, EU Grant No. FP7-216025 BIND, and SFI Grant No. RFP 06/RFP/ENE039 for funding this work. The work of J.K.V. at Sungkyunkwan University was supported by the World Class University program through the National Research Foundation of Korea, funded by the Ministry of Education, Science and Technology (Grant No. R32-2008-000-10204-0).
[1] W. Maier and A. Saupe, Z. Naturforsch. 13, 564 (1958); 14, 882 (1959); 15, 287 (1960).

[2] P. G. de Gennes and J. Prost, The Physics of Liquid Crystals (Clarendon, Oxford, 1993).

[3] M. J. Freiser, Phys. Rev. Lett. 24, 1041 (1970).

[4] J. P. Straley, Phys. Rev. A 10, 1881 (1974).

[5] R. Alben, Phys. Rev. Lett. 30, 778 (1973).

[6] C. S. Shih and R. Alben, J. Chem. Phys. 57, 3057 (1972).

[7] G. R. Luckhurst and S. Romano, Mol. Phys. 40, 129 (1980).

[8] F. Biscarini, C. Chiccoli, P. Pasini, F. Semeria, and C. Zannoni, Phys. Rev. Lett. 75, 1803 (1995).

[9] M. P. Allen, Liq. Cryst. 8, 499 (1990).

[10] J. Pelaez and M. R. Wilson, Phys. Rev. Lett. 97, 267801 (2006).

[11] R. Berardi, L. Muccoli, S. Orlandi, M. Ricci, and C. Zannoni, J. Phys.: Condens. Matter 20, 463101 (2008).

[12] A. Eremin, S. Diele, G. Pelzl, H. Nadasi, W. Weissflog, J. Salfetnikova, and H. Kresse, Phys. Rev. E 64, 051707 (2001).

[13] B. K. Sadashiva, R. A. Reddy, R. Pratibha, and N. V. Madhusudana, J. Mater. Chem. 12, 943 (2002).

[14] D. Pociecha, E. Gorecka, M. Cepic, N. Vaupotic, K. Gomola, and J. Mieczkowski, Phys. Rev. E 72, 060701 (2005).

[15] M. Nagaraj, Y. P. Panarin, J. K. Vij, C. Keith, and C. Tschierske, Appl. Phys. Lett. 97, 213505 (2010).
[16] Y. P. Panarin, M. Nagaraj, J. K. Vij, C. Keith, and C. Tschierske, Euro. Phys. Lett. 92, 26002 (2010).

[17] D. Pociecha, M. Cepic, E. Gorecka, and J. Mieczkowski, Phys. Rev. Lett. 91, 185501 (2003).

[18] K. Gomola, L. Guo, D. Pociecha, F. Araoka, K. Ishikawa, and H. Takezoe, J. Mater. Chem. 20, 7944 (2010).

[19] R. A. Reddy, Ch. Zhu, R. Shao, E. Korblova, T. Gong, Yo. Shen, E. Garcia, M. A. Glaser, J. E. Maclennan, D. M. Walba, and N. A. Clark, Science 332, 72 (2011).

[20] Y. P. Panarin, M. Nagaraj, S. Sreenilayam, J. K. Vij, A. Lehmann, and C. Tschierske, Phys. Rev. Lett. 107, 247801 (2011).

[21] M. V. Gorkunov, M. A. Osipov, A. Kocot, and J. K. Vij, Phys. Rev. E 81, 061702 (2010).

[22] M. F. Vuks, Opt. Spektrosk. 20, 651 (1966).

[23] W. Kuczynski, B. Zywucki, and J. Matecki, Mol. Cryst. Liq. Cryst. 381, 1 (2002).

[24] I. Haller, Prog. Solid State Chem. 10, 103 (1975).

[25] C. Keith, M. Prehm, Y. P. Panarin, J. K. Vij, and C. Tschierske, Chem. Commun. 46, 3702 (2010).

[26] S. Sreenilayam, M. Nagaraj, Y. P. Panarin, J. K. Vij, A. Lehmann, and C. Tschierske, Molec. Cryst. Liq. Cryst. 553, 133 (2012). 